\title{
The alveolar immune cell landscape is dysregulated in checkpoint inhibitor pneumonitis
}

Karthik Suresh, ${ }^{1}$ Jarushka Naidoo, ${ }^{2,3}$ Qiong Zhong, ${ }^{1}$ Ye Xiong, ${ }^{1}$ Jennifer Mammen, ${ }^{4}$ Marcia Villegas de Flores, ${ }^{5}$ Laura Cappelli, ${ }^{5}$ Aanika Balaji, ${ }^{2}$ Tsvi Palmer, ${ }^{1}$ Patrick M. Forde, ${ }^{2,3}$ Valsamo Anagnostou, ${ }^{2,3}$ David S. Ettinger, ${ }^{2}$ Kristen A. Marrone, ${ }^{2,3}$ Ronan J. Kelly, ${ }^{2,3}$ Christine L. Hann, ${ }^{2,3}$ Benjamin Levy, ${ }^{2,3}$ Josephine L. Feliciano, ${ }^{2,3}$ Cheng-Ting Lin, ${ }^{6}$ David Feller-Kopman, ${ }^{1}$ Andrew D. Lerner, ${ }^{1}$ Hans Lee, ${ }^{1}$ Majid Shafiq, ${ }^{1}$ Lonny Yarmus, ${ }^{1}$ Evan J. Lipson, ${ }^{3,4}$ Mark Soloski, Julie R. Brahmer, ${ }^{2,3}$ Sonye K. Danoff,' and Franco D'Alessio'

'Division of Pulmonary Critical Care Medicine, and '2Department of Oncology, Johns Hopkins University School of Medicine, Baltimore, Maryland, USA. ${ }^{3}$ Bloomberg-Kimmel Institute for Cancer Immunotherapy at Johns Hopkins University, Baltimore, Maryland, USA. ${ }^{4}$ Division of Endocrinology, ${ }^{5}$ Division of Rheumatology, and ${ }^{6}$ Department of Radiology, Johns Hopkins University School of Medicine, Baltimore, Maryland, USA.

BACKGROUND. Checkpoint inhibitor pneumonitis (CIP) is a highly morbid complication of immune checkpoint immunotherapy (ICI), one which precludes the continuation of ICI. Yet, the mechanistic underpinnings of CIP are unknown.

METHODS. To better understand the mechanism of lung injury in CIP, we prospectively collected bronchoalveolar lavage (BAL) samples in ICl-treated patients with $(n=12)$ and without CIP $(n=6)$, prior to initiating first-line therapy for CIP (high-dose corticosteroids). We analyzed BAL immune cell populations using a combination of traditional multicolor flow cytometry gating, unsupervised clustering analysis, and BAL supernatant cytokine measurements.

RESULTS. We found increased BAL lymphocytosis, predominantly CD4+ T cells, in patients with CIP. Specifically, we observed increased numbers of BAL central memory T cells, evidence of type I polarization, and decreased expression of cytotoxic T lymphocyte-associated protein 4 and programmed cell death protein 1 in BAL Tregs, suggesting both activation of proinflammatory subsets and an attenuated suppressive phenotype. CIP BAL myeloid immune populations displayed enhanced expression of IL-1 $1 \beta$ and decreased expression of counterregulatory interleukin-1 receptor antagonist. We observed increased levels of T-cell chemoattractants in the BAL supernatant, consistent with our proinflammatory, lymphocytic cellular landscape.

CONCLUSION. We observe several immune cell subpopulations that are dysregulated in CIP, which may represent possible targets that could lead to therapeutics for this morbid immune-related adverse event.

FUNDING. NIH, Department of Defense, and the Bloomberg Kimmel Institute for Cancer Immunotherapy.

\section{Introduction}

With recent clinical trials demonstrating clear efficacy for immunotherapy in patients with locally advanced and advanced-stage non-small-cell lung cancer (NSCLC) as well as other tumors, the use of immune checkpoint inhibitors (ICIs) for the treatment of NSCLC has rapidly increased (1-3), becoming the standard of care. ICIs, however, are associated with a constellation of toxicities termed immune-related adverse events (irAEs). These toxicities include arthritis, colitis, endocrinopathies, and lung injury; the last is termed checkpoint inhibitor pneumonitis (CIP) $(4,5)$. Clinically, patients with CIP present with acute to subacute onset of dys-

Authorship note: KS and JN contributed equally to this work Conflict of interest: The authors have declared that no conflict of interest exists. Copyright: @ 2019, American Society for Clinical Investigation.

Submitted: March 27, 2019; Accepted: July 9, 2019; Published: September 4, 2019 Reference information: J Clin Invest. 2019;129(10):4305-4315.

https://doi.org/10.1172/JCl128654. pnea, hypoxemia, and pulmonary infiltrates similar to that seen in patients with lung injury from acute respiratory distress syndrome (6). Although CIP can result in high morbidity, it was previously thought to be an uncommon complication of ICI therapy, with an incidence of around $3 \%$ to $5 \%(7,8)$ based on clinical trial data. Recent evidence from our group and others suggests, however, that the occurrence of CIP may be higher in real-world settings (9, 10). For instance, using a multidisciplinary, standardized approach (11), we recently observed an incidence of $19 \%$ in a cohort of 205 patients with NSCLC treated with ICI (12). In addition, we also observed an association between CIP development and increased mortality rates in patients with NSCLC treated with ICI (13).

Despite the rising incidence of CIP and its association with increased mortality, the current paradigms for diagnosis and treatment of CIP are largely based on anecdotal evidence, primarily because fundamental knowledge of CIP pathobiology is lacking (14). CIP is diagnosed by the presence of compatible symptoms (shortness of breath, hypoxia, cough), new radiographic infil- 

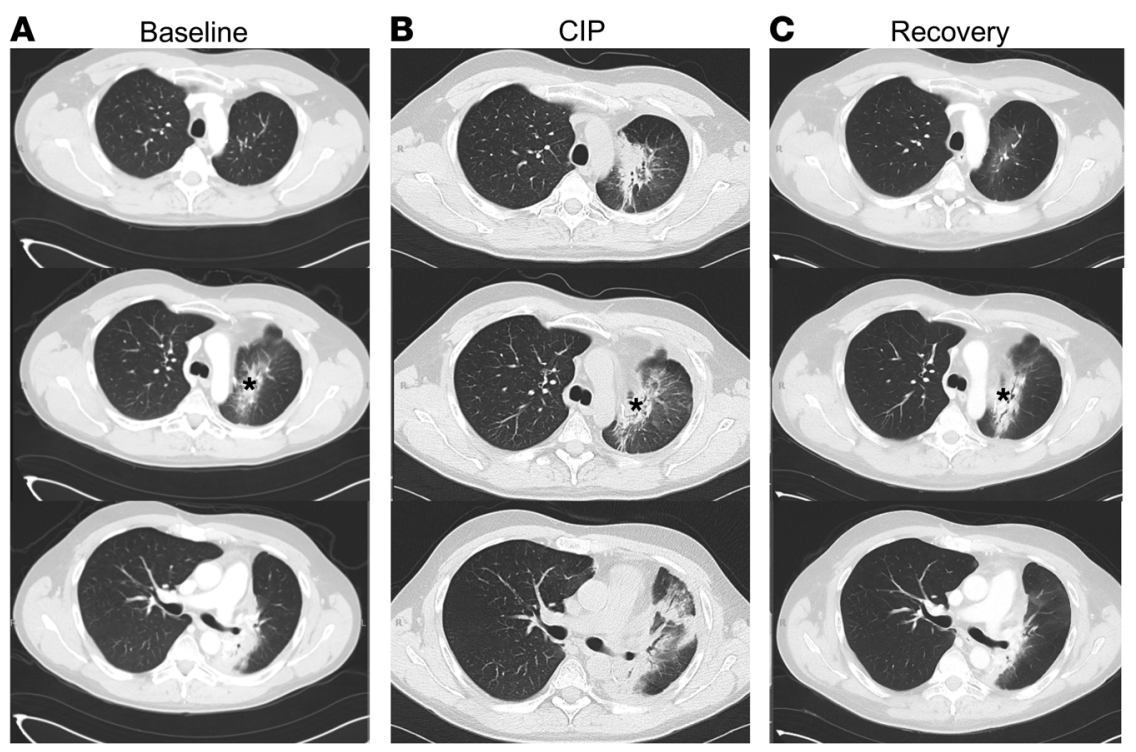

Figure 1. Radiographic presentation of CIP. Representative computed tomography images of an ICI-treated NSCLC patient (A) prior to development of CIP, (B) at the time of CIP diagnosis, and (C) after 3 weeks of steroid treatment. *Denotes area of preexisting post-radiotherapy changes that were stable before initiation of ICI.

trates, which can be either unilateral or bilateral $(15,16)$, typically with ground glass and consolidative components (Figure 1), and the exclusion of infectious etiologies (with sputum cultures or bronchoalveolar lavage [BAL]). There are currently no diagnostic biomarkers for CIP, so the diagnosis remains largely one of exclusion. Once diagnosed, clinical severity is used to determine CIP grade (Supplemental Table 1; supplemental material available online with this article; https://doi.org/10.1172/JCI128654DS1). For CIP grade 2 (i.e., symptomatic patients with compatible radiographic infiltrates) and higher, ICI therapy is immediately discontinued, and empiric high-dose steroids are initiated. More targeted, disease-specific therapy is not instituted as first-line treatment for CIP in part because there are currently no available data on the mechanism of lung injury in CIP. Due to the lack of diagnostic and therapeutic options, patients diagnosed with CIP are typically also not eligible for further ICI; this is particularly disadvantageous in individuals with ongoing tumor response.

As part of a multidisciplinary immune-related toxicity (irTox) team (11) engaged in diagnosis, management, and study of irAEs following ICI therapy, we prospectively collected BAL fluid (BALF) specimens from patients treated with ICI who have no evidence of CIP as well as those with suspected CIP. Clinical, laboratory, and radiographic data of patients suspected of having CIP were subsequently reviewed by the irTox team, and a determination was made as to whether the presenting symptoms were due to CIP or another etiology. Using these specimens, we performed multiparametric flow cytometric analysis on BALF samples to better understand the landscape of immune dysregulation in CIP. In part

Figure 2. Study design and participating patients. Consort diagram showing study enrollment and adjudication of patients into control and CIP groups. *Pertinent clinical, radiographic, laboratory and microbiologic (including BAL culture when available) data were reviewed by the immune-related toxicity (irTox) team before and (in cases of suspected CIP) after bronchoscopy. At both time points, patients with suspected CIP with an alternative etiology for symptoms were excluded from the CIP group $(n=2)$. ICI, immune checkpoint inhibitor; VATS, video-assisted thoracic surgery; CIP, checkpoint inhibitor pneumonitis. due to the lack of available data on the biology of lung injury in CIP, we utilized unbiased clustering analytic techniques to examine our flow cytometric results. Such approaches have the advantage of detecting changes in small cell populations that may otherwise be excluded with manual gating. Importantly, the control group comprised patients who also received ICI but did not exhibit any clinical evidence of CIP at the time of bronchoscopy.

\section{Results}

BAL lymphocytosis is a hallmark for CIP. Study design as well as baseline clinical characteristics for the patients enrolled in this observational study are shown in Figure 2 and Table 1, respec-

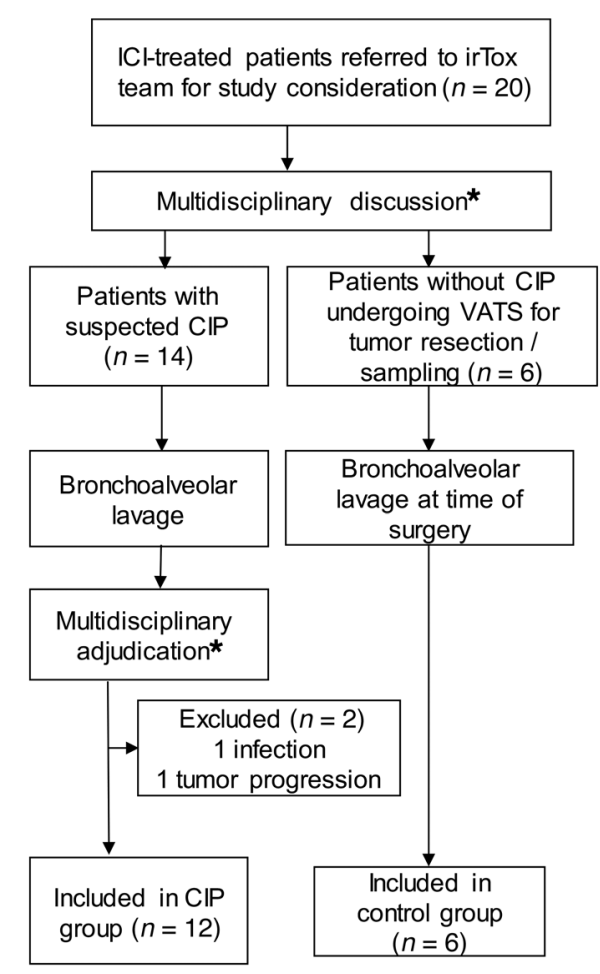


Table 1. Baseline characteristics

\begin{tabular}{|c|c|c|c|}
\hline & No CIP $(n=6)$ & $\operatorname{CIP}(n=12)$ & $P$ value \\
\hline Median age - yr & $60(55-65)$ & $71(65-77)$ & \\
\hline Female sex - no. (\%) & $1(16)$ & $4(33)$ & 0.8 \\
\hline Race - no. (\%) & & & 0.72 \\
\hline White & $4(66)$ & $10(83)$ & \\
\hline African American & $1(17)$ & $1(8)$ & \\
\hline Other & $1(17)$ & $1(8)$ & \\
\hline Smoking - no. (\%) & & & 1 \\
\hline Former & $5(83)$ & $9(72)$ & \\
\hline Never & $1(17)$ & $3(27)$ & \\
\hline Tumor histology - no. (\%) & & & 0.27 \\
\hline Melanoma & $1(17)$ & $1(8)$ & \\
\hline NSCLC & $4(66)$ & $11(92)$ & \\
\hline Other ${ }^{A}$ & $1(17)$ & 0 & \\
\hline Prior chemotherapy - no. (\%) & $5(83)$ & $9(75)$ & 1 \\
\hline Radiation & $4(66)$ & $4(36)$ & 0.3 \\
\hline ICl agent - no. (\%) & & & 0.54 \\
\hline Nivolumab & $2(33)$ & $7(33)$ & \\
\hline Pembrolizumab & $1(17)$ & $4(33)$ & \\
\hline Other ${ }^{B}$ & $1(17)$ & $1(8)$ & \\
\hline Combination ICI & $2(33)$ & $5(18)$ & \\
\hline \multicolumn{4}{|l|}{ Comorbid pulmonary conditions } \\
\hline COPD & $3(50)$ & $3(27)$ & 0.6 \\
\hline ILD & 0 & 0 & - \\
\hline Pulmonary embolism & $1(17)$ & $2(16)$ & 1 \\
\hline
\end{tabular}

${ }^{A}$ Basal cell carcinoma (skin). ${ }^{B}$ Durvalumab. COPD, chronic obstructive pulmonary disease; ICI, immune checkpoint inhibitor; ILD, interstitial lung disease; NSCLC, non-small-cell lung cancer.

tively. Clinical grade, management, and outcomes data for the 12 patients with CIP are presented in Supplemental Table 2. We first manually counted BAL cell differentials in a subset of control and CIP samples. We found a relative increase in lymphocytes with a concomitant decrease in monocytes in CIP (Supplemental Figure 2) compared with patients without CIP. Notably, BAL neutrophils were not abundant in patients with CIP. To further characterize subsets of BAL immune cells, we performed multiparametric flow cytometric analysis using optimized T cell and monocyte panels (Supplemental Table 3). We initially analyzed these data using traditional gating methods, and similar to our manual cell differentials, found an increase in the percentage of $\mathrm{T}$ lymphocytes in patients with CIP (Figure 3). Specifically, we found an increase in $\mathrm{CD}^{+} \mathrm{CD}^{+}$cells (Figure $3 \mathrm{~A} ; P=0.04$ ) and a possible association with increased $\mathrm{CD}^{+} \mathrm{CD}^{+}$cells (Figure 3B; $P=$ 0.073). We also noted a decrease in monocytes, specifically CD3-CD19-CD14 ${ }^{+}$cells (Figure 3C; $P=0.04)$. We did not observe any differences in the percentage of Tregs $\left(\mathrm{CD}^{+} \mathrm{CD} 4^{+} \mathrm{CD} 127^{\text {lo }}\right.$ $\mathrm{CD} 25^{+} \mathrm{Foxp}^{+}$) among patients who were CIP+ and CIP- (data not shown).
Unsupervised clustering reveals differential T cell subpopulations in CIP. To understand immune cell subpopulations in our samples in more granular detail, we next turned to unsupervised clustering analysis. The total numbers of cells per condition used for our unsupervised analyses are shown in Supplemental Table 4. We represent the results of our clustering analysis using star charts. As shown in Supplemental Figure 1, groups of cells that share similar cytokine profiles are identified as a node and represented by a circle. The diameter of the circle reflects the number of cells present within that subpopulation. The cell surface or intracellular mean fluorescence intensity (MFI) for each fluorophore is expressed as a wedge within the circle; the radius of the wedge segment represents the expression level of that particular marker. For instance, in Supplemental Figure 1B, a node with very high PD-1, CD45RA, and CD127 expression is shown. Topologically, nodes are arranged by similarity to each other in a cluster map (Supplemental Figure 1C). Cell subsets occupy distinct areas within a map; for instance, in the $\mathrm{T}$ cell cluster map, as expected, $\mathrm{CD} 4^{+}$and $\mathrm{CD} 8^{+}$cells are clustered together in opposite ends because they are very distinct from each other (Supplemental Figure 1, D and E).

In control, unstimulated $\mathrm{T}$ cells, we observed clustering around 2 cell populations: $\mathrm{CD} 4^{+}$cells with high $\mathrm{PD}-1$ expression (Figure 4A) and CD8 ${ }^{+}$cells with moderate PD-1 expression (Figure 4A). Unstimulated CIP samples exhibited increased CD8 ${ }^{+}$cell populations compared with unstimulated controls (Figure 4B) as well as a local shift in $\mathrm{CD}^{+}$Treg populations (Figure $4 \mathrm{~B}$ ), as discussed in more detail in the information to follow.

To better understand the specific T cell subsets that were up/ downregulated in patients with CIP, we examined the differential cluster map of $\mathrm{T}$ cell subsets, which highlights only clusters where the magnitude of difference between groups was greater than 95\%. As shown in Figure 5, in CIP, we observed a significant increase in $\mathrm{CD} 4{ }^{+} \mathrm{CD} 45 \mathrm{RA}^{+} \mathrm{CD} 25^{-}$cells that also expressed CD $62 \mathrm{~L}$. Because this cytokine profile resembled that of central memory $\mathrm{T}$ cells (Tcms), a non-Treg (i.e., conventional) T cell subpopulation characterized by high CD62L and low CD45RA expression, we performed manual gating for Treg and non-Treg subpopulations (Supplemental Figure 3) and observed a significantly higher per-
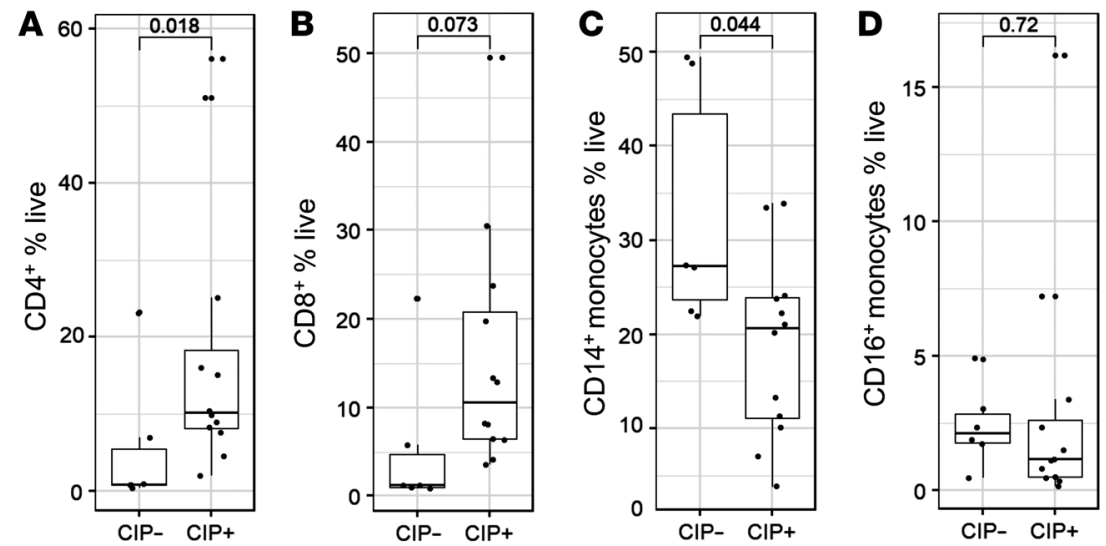

Figure 3. BAL lymphocytosis in patients with CIP. Scatter plots showing number of (A) CD4+ (B) $\mathrm{CD}^{+}$, (C) CD14+, and (D) CD16+ T cells (A and B) and monocytes (C and D), respectively, in control and CIP samples. $n=6$ (CIP-), 12 (CIP+). Comparisons between groups performed using Mann-Whitney test. 

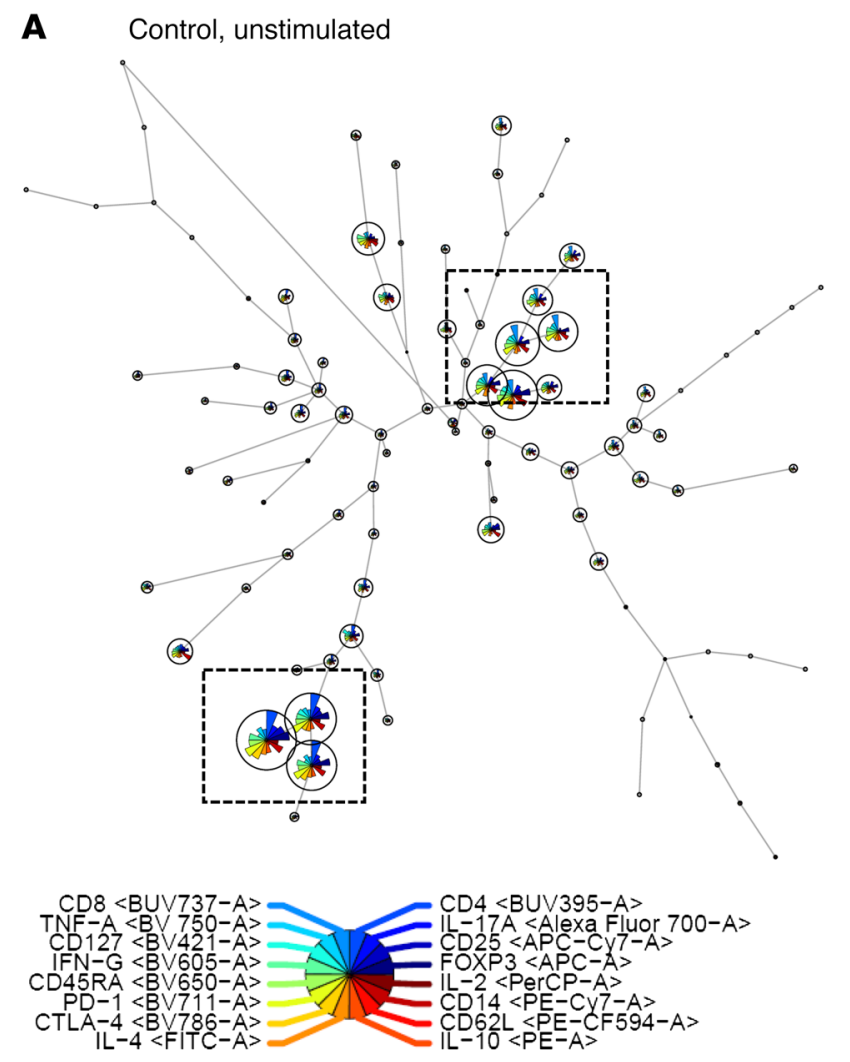

centage of Tcm in CIP samples $(P=0.01)$. As mentioned earlier, we observed a shift in $\mathrm{CD}^{+} \mathrm{FoxP}^{+}$cells between unstimulated control and CIP cluster maps. Closer examination of these clusters revealed that while clusters of $\mathrm{PD}-1^{\mathrm{lo}} \mathrm{CTLA}-4^{\text {lo }}$ Tregs were similarly expressed in both CIP and controls, a subpopulation of Tregs with high PD-1 and CTLA-4 expression was only seen in controls, and these effector molecules were downregulated in alveolar Tregs in CIP (Figure 5). Compared with controls, multiple CD8 ${ }^{+}$TNF- $\alpha^{\text {hi }}$ subpopulations were upregulated at baseline in CIP (Figure 5). Ex vivo stimulation of CIP samples polarized T cells toward a type 1 phenotype with increased TNF- $\alpha$ and IFN- $\gamma$ production across multiple cell subsets with varying degrees of CD8 expression; these cell populations were not increased in control cells following stimulation (Supplemental Figure 4).

In summary, these findings suggest multiple dysregulated $\mathrm{T}$ cell subsets in patients with CIP. At baseline, we observe in CIP: (a) increased Tcms, (b) loss of PD- $1^{\text {hi }} /$ CTLA $-4^{\text {hi }}$ CD $4^{+}$Tregs and (c) upregulation of proinflammatory (i.e., TNF- $\alpha^{\text {hi }}$, IFN- $\left.\gamma^{\text {hi }}\right) \mathrm{CD}^{+}$ cells. With stimulation, we observe an increase in numbers of $\mathrm{CD} 8^{+} \mathrm{TNF}-\alpha^{\text {hi }}$ subsets and the amount of TNF- $\alpha$ expression in stimulated CIP samples compared with controls.

Upregulation of IL-1 $\beta^{\text {hi }}$ monocytes in CIP and IL-1RA-expressing $B$ cells in controls. Next, we sought to examine population differences in non-T (i.e., CD3-) cells. Similar to our T cell analyses, we represented the results in cluster maps where the distinct cell populations (e.g., CD $14^{+}$monocytes, CD $16^{+}$monocytes, B cells) occupy various regions within the map (Supplemental Figure 5). We observed clear differences between unstimulated control and CIP samples (Figure 6). As shown in Figure 6, A and B, and in closer detail in Figure 7, two reciprocal populations were upregulated
B

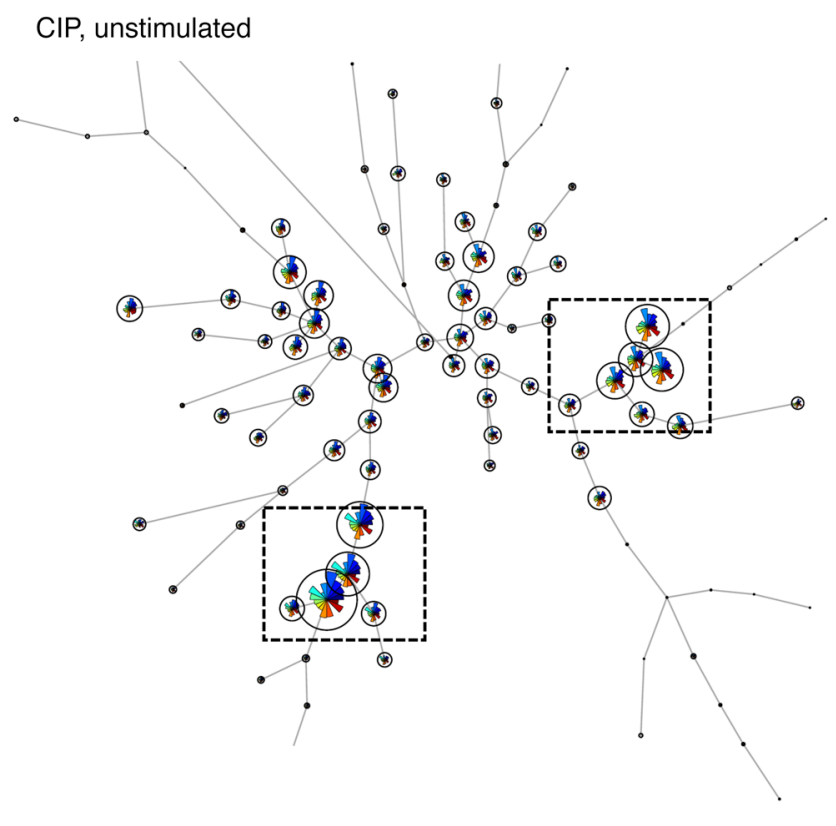

Figure 4. T cell populations in CIP. Unsupervised clustering of T cells in BALF samples of patients without (control, $n=6)$ and with CIP $(n=12)$. Cluster maps showing distribution of T cell subpopulations in (A) unstimulated controls and (B) unstimulated CIP. Within each cluster map, larger cell populations distinct to that particular condition are highlighted (square boxes).

in controls and CIP, respectively. In controls, we observed a large increase in several clusters corresponding to IL-1RA-expressing $\mathrm{CD}^{+} 6^{+} \mathrm{B}$ cells $\left(\mathrm{CD} 19^{+}\right)$. While this cluster was downregulated in CIP, a different cluster of IL- $1 \beta^{\text {hiTNF- }} \alpha^{\text {hi }} C D-11 b^{\text {hi }}$ myeloid cells (CD19-, CD14 ${ }^{\text {int }} /$ CD16 $\left.{ }^{\text {int }}\right)$ was significantly upregulated in CIP. Similar to our $\mathrm{T}$ cell analysis, we confirmed the presence of a TNF- $\alpha^{\text {hi }} I L-1 \beta^{\text {hi }} C D 11 b^{\text {hi }}$ population in CIP samples with manual gating (Supplemental Figure 6). Unlike T cells, we did not observe significant differences in cluster profiles between unstimulated and stimulated cells either in the control or CIP condition (Supplemental Figure 7).

We also compared the subpopulations identified previously as being significantly different in controls or CIP to the results of a meta-clustering analysis, to determine whether the subpopulations selected to be differentially upregulated in our prior analyses were also identified as distinct populations using an autogating strategy. As shown in Supplemental Figure 8, meta-clustering identified the clusters previously examined in our T cell and monocyte/B cell cluster maps (Figure 4 and Figure 6) as distinct subpopulations.

Upregulation of lymphocyte chemoattractants in the BALF of patients with CIP. To determine whether BALF cytokines were promoting the cellular phenotypes observed in our flow cytometry data, we measured key cytokines in the cell-free BAL supernatant (Figure 8, A-C, and Supplemental Table 5). Surprisingly, despite observing an increased number of IL-1 $1 \beta^{\text {hi }}$ cells in our flow analysis, we observed decreased levels of IL-1 $1 \beta$ in CIP BAL supernatants. We observed no differences in TNF- $\alpha$ levels, but discovered increased levels of the type 1 skewing cytokine IL-12p40. We also measured levels of cytokines involved in the recruitment of inflammatory cells to the alveolus. We observed lower levels of 
Cluster increased in controls

Cluster increased in CIP

\section{i. $\quad \mathrm{CD}^{+} \mathrm{FoxP}^{10} \mathrm{CD} 25^{-}$} CD62L hi CD45RA ${ }^{\text {lo }}(\mathbf{T c m}$ increased in $\mathrm{CIP}$ )
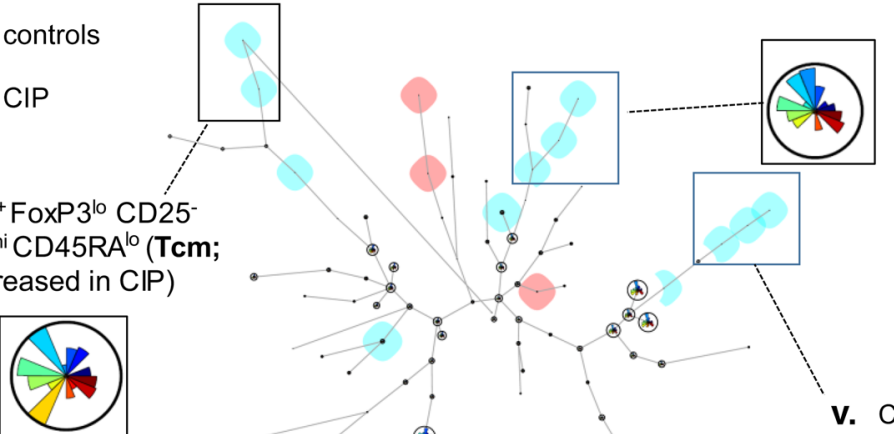

vi.

CD8 ${ }^{+}$TNF- $\alpha^{\text {hi }}$ IFN- $\gamma^{\text {hi }}$ PD- $1^{\text {mod }}$ (increased in CIP)

ii. $\quad C D 4^{+} F$ oxP3 $3^{+} P D-1^{\text {hi }}, C T L A-4^{\text {hi }}$
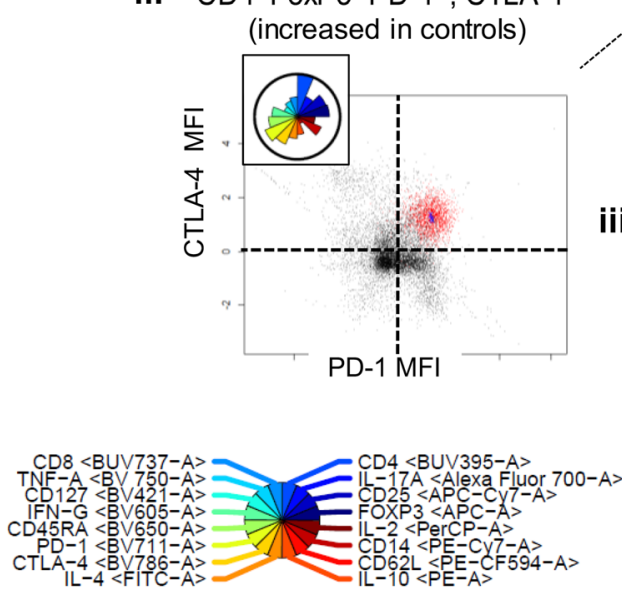

iii. $\mathrm{CD}^{+} \mathrm{FoxP}^{+} \mathrm{PD}-1^{\text {lo }} \mathrm{CTLA}-4^{\text {lo }}$ (similarly present in CIP and controls)

v. $C D 8^{+} T N F-\alpha^{\text {hi }} I F N-\gamma^{\text {hi } P D}-1^{\text {hi }}$ (increased in CIP)
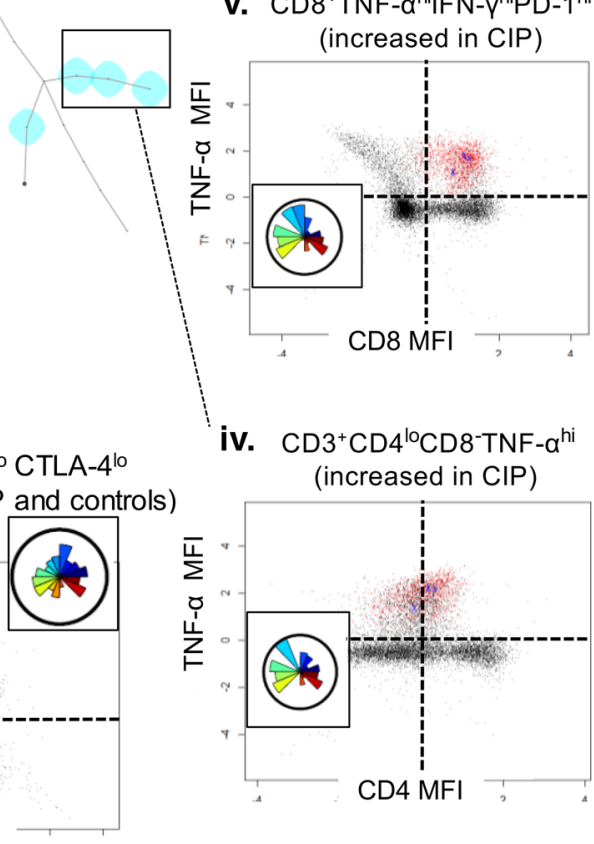

iv. $\mathrm{CD}^{+} \mathrm{CD} 4^{\mathrm{lo}} \mathrm{CD} 8^{-} \mathrm{TNF}-\mathrm{\alpha}^{\text {hi }}$
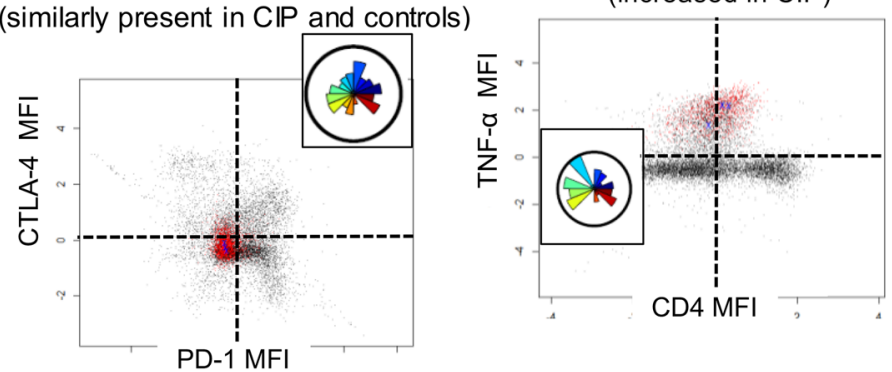

Figure 5. Abnormal T cell subsets in CIP. Differential cluster map (center) shows clusters where the number of cells within the cluster were increased by $95 \%$ in controls (red, $n=6$ ) or CIP (cyan, $n=12$ ). Cytokine profile (inset) and scatter plot of relevant cytokines showing MFI in the selected clusters (red) compared with MFI across all clusters (black) in (counterclockwise): (i) CD4+FoxP3 ${ }^{\text {lo }} C D 25-C D 62 L^{\text {hi }} C D 45 R A^{\text {lo }}$ cluster increased in CIP; (ii) PD-1 ${ }^{\text {hi }}$ CTLA-4 $4^{\text {hi }}$ clusters of Tregs increased in controls, scatter plot showing PD-1/CTLA-4 MFI in selected clusters; (iii) similar (i.e., $<95 \%$ difference) expression of PD-1 $1^{10} \mathrm{CTLA}-4^{10}$ Treg clusters in CIP and controls, scatter plot showing PD-1/CTLA-4 MFI in selected clusters; (iv) a CD3+CD4 ${ }^{\circ}$ CD8-TNF- $\alpha^{\text {hi }}$ population increased in CIP, scatter plot showing

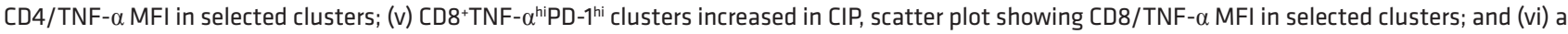
second set of CD8 ${ }^{+}$TNF- $\alpha^{\text {hi }}$ clusters increased in CIP.

IL-8, the classical neutrophil chemoattractant, in CIP. Although no differences were seen in levels of monocyte chemoattractant proteins 1 or 4, we observed lower levels of macrophage inflammatory protein-3 $\alpha$ (MIP-3 $\alpha$ ), a significant increase in levels of the IFN- $\gamma$-induced protein 10 (IP-10, or CXCL-10) and a trend toward increased levels of $\mathrm{T}$ cell chemoattractant protein TARC (also known as CCL17; $P=0.06$ ).

\section{Discussion}

In this study, we describe multiple baseline and functional abnormalities in both lymphoid and myeloid alveolar cell types in patients who developed CIP. These abnormalities involve both upregulation of proinflammatory subsets and downregulation of the counterregulatory antiinflammatory process in both $\mathrm{T}$ cells and myeloid cells (Figure 9). In healthy adults, the BAL is composed primarily of macrophages (>85\%) and lymphocytes $(10 \%)$ (17). These percentages are similar to the pattern seen in our con- trol samples (i.e., patients who received ICI but did not have CIP at the time of bronchoscopy), suggesting that ICI therapy alone does not appear to significantly alter the alveolar immune cell pattern. In contrast, we observed lymphocytosis of greater than $20 \%$ in most of our $\mathrm{CIP}^{+}$BAL samples. BAL lymphocytosis has been reported in other conditions such as sarcoidosis, hypersensitivity pneumonitis, cryptogenic organizing pneumonia, nonspecific interstitial pneumonia, and radiation pneumonitis. Our finding of lymphocytosis in the BALF of patients with CIP argues for the use of BAL cell count differentials and flow cytometry for $\mathrm{CD}^{+} / \mathrm{CD}^{+}$cells as part of the clinical evaluation scheme during BAL in patients with suspected CIP. As no biomarker currently exists for this disease, this discovery represents a translational application of our current findings.

Our unbiased clustering approach identified several subpopulations of $\mathrm{T}$ cells that are likely to be playing key roles in the pathobiology of CIP. First, $\mathrm{CD} 4^{+}$central memory subsets (Tcms, 


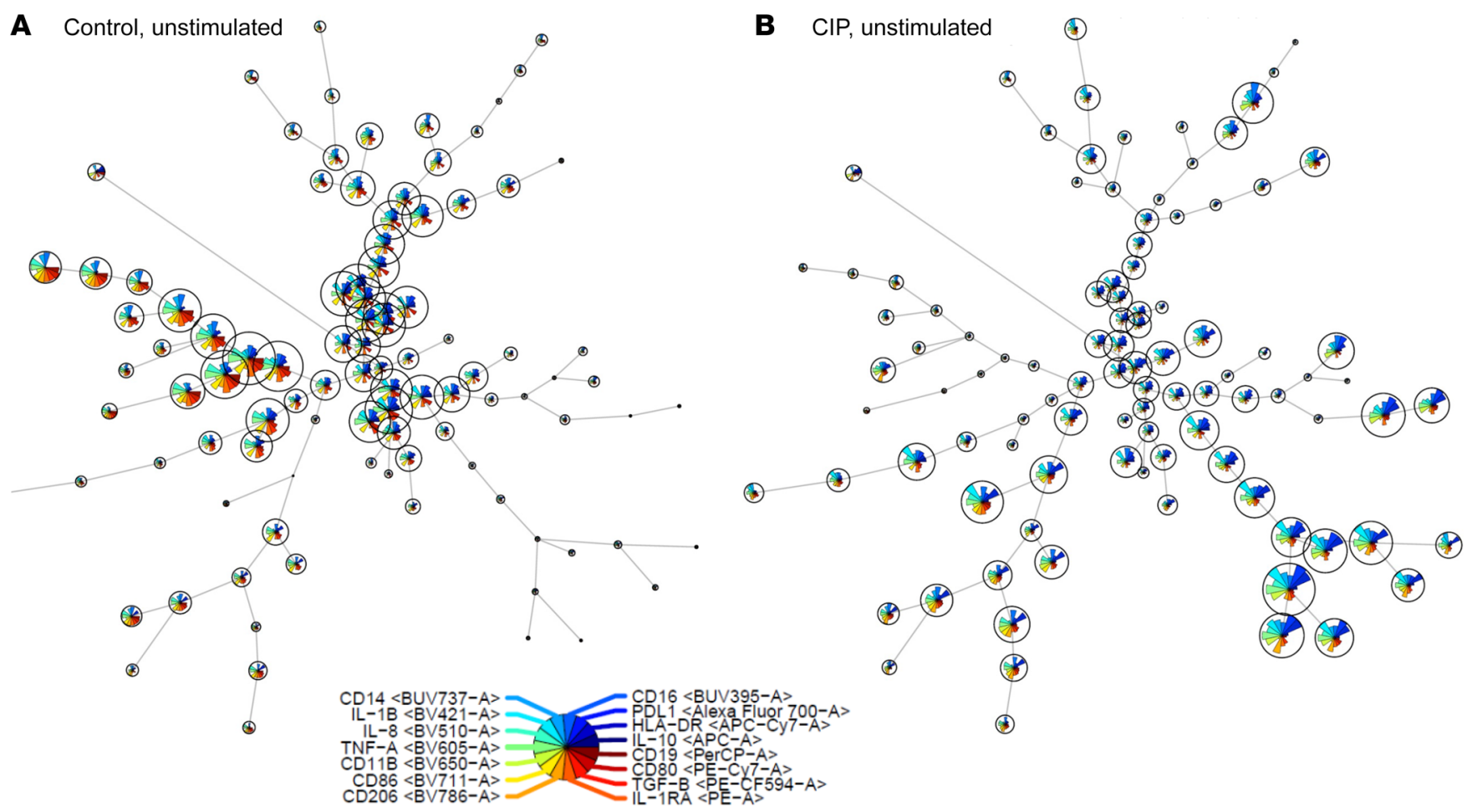

Figure 6. Monocyte populations in CIP. Unsupervised clustering of non-T cells (singlet, live, CD3 $^{-}$) in BALF samples of patients without (control, $\left.n=6\right)$ and with CIP $(n=12)$. Cluster maps showing distribution of myeloid subpopulations in $(\mathbf{A})$ unstimulated controls and $(\mathbf{B})$ unstimulated CIP.

$\left.\mathrm{CD}^{+}{ }^{+} \mathrm{CD} 45 \mathrm{RA}^{-} \mathrm{CD} 62 \mathrm{~L}^{+}\right)$were increased in CIP. Tcms have been shown to be more resistant to steroid-induced apoptosis than other conventional $\mathrm{T}$ cells, such as effector memory $\mathrm{T}$ cells. Moreover, $\mathrm{CD}_{62} \mathrm{~L}^{+}$cells play an important role in adhesion to inflammatory sites and can perpetuate injury (18). Increased Tcm in CIP might explain why some patients fail high-dose steroid therapy. We recently reported steroid-refractory disease in up to $40 \%$ of patients with CIP in our cohort (10); from a lung injury standpoint, this feature of CIP is unique compared with other lymphocytic pneumonitides, which generally tend to be steroid responsive. The incidence of CIP is significantly higher in patients with underlying NSCLC than other cancers, and we have shown (5) that within patients with NSCLC, tumor histology further stratifies CIP incidence and risk. These findings, coupled with our current data, suggest that Tcm could be responding to tumor-specific antigens. $\mathrm{T}$ cell receptor sequencing of the $\mathrm{T}$ cell subsets in CIP samples will be useful in this regard. Second, a subpopulation of $\mathrm{CD} 4^{+}$cells skewed toward a type I phenotype with high IFN- $\gamma$ and TNF- $\alpha$ production is upregulated in CIP. Type I lymphocytes have been linked to several lung diseases including sarcoidosis, hypersensitivity pneumonitis, and lung allograft rejection (19-21). Thus, the combination of "sticky" lung CD4 ${ }^{+} \mathrm{T}$ cells (i.e., CD62 $\mathrm{L}^{+}$ $\mathrm{CD} 4^{+}$cells) and type I skewing may be synergistically contributing to lung injury seen in patients with CIP.

Third, we observed decreased CTLA- 4 and PD-1 expression within Treg (i.e., FoxP3 ${ }^{+}$) populations, suggesting an attenuated Treg suppressive phenotype. One explanation for our findings is that, in CIP, loss of Treg suppression may be promoting exuberant Th1 T cell responses. We have shown that alveolar Tregs play a pivotal role orchestrating resolution of lung inflammation and are present in humans with lung injury (22), while others have shown that $\mathrm{PD}-1^{+}$Tregs are more suppressive to control $\mathrm{CD}^{+} \mathrm{T}$ cells (23). In addition to PD-1, the lack of CTLA-4 may further impair Treg ability to control conventional T cell (such as Tcm) and macrophage proinflammatory responses (24). Overall, our findings suggest highly activated alveolar $\mathrm{T}$ cells with loss of a regulatory, antiinflammatory Treg suppressive phenotype contributing to unchecked immune dysregulation seen in CIP.

Interestingly, while we observed decreased numbers of CD14 ${ }^{+}$ monocytes based on traditional gating methods, our clustering data show additional dramatic shifts in myeloid populations between controls and patients with CIP, such as a significant increase in CD11b ${ }^{\text {hi }} I L-1 \beta^{\text {hi }}$, myeloid cells with varying degrees of CD14/CD16 expression. This is accompanied by a loss of IL-1RA $\mathrm{RD}^{+} \mathrm{C}^{+}$cells in patients with CIP, reflected in the cluster maps as a relative upregulation of these cells in controls. These findings suggest that an imbalance in IL-1 signaling, along with overexuberant TNF- $\alpha$ signaling may be contributing to the pathobiology of lung injury in patients with CIP. The concomitant presence of increased Tcms, as discussed earlier, may also serve to augment $\mathrm{T}$ cell and monocyte inflammation.

Our BALF cytokine results also point toward a proinflammatory, chemoattractant cytokine milieu. Interestingly, we observed a decrease in soluble IL- $1 \beta$, while an increase in IL- $1 \beta$-expressing monocyte subsets was observed in flow cytometry. The dynamics of IL- $1 \beta$ production and release is complex, however, and thought to be related to the strength of the inflammatory stimulus (25). Thus, one possibility is that, in CIP, the underlying source of inflammation promotes IL-1 $\beta$ translation and endosomal storage, but not membrane release. Another possibility is that soluble 


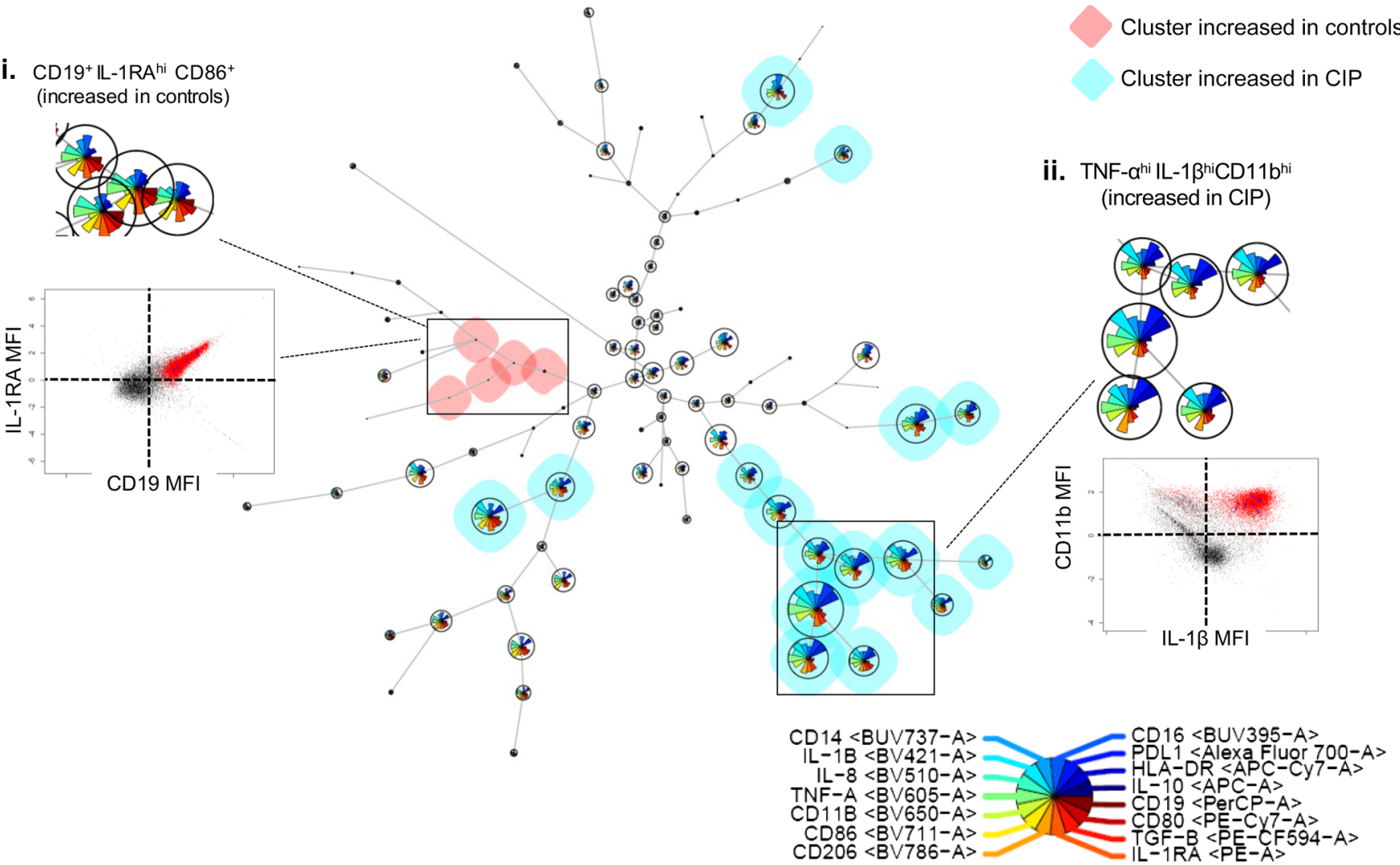

Figure 7. Abnormal monocyte subsets in CIP. Differential cluster map of myeloid cells showing clusters that are increased by at least $95 \%$ between unstimulated controls $(n=6)$ and unstimulated CIP $(n=12)$ samples. Cytokine profiles and scatter plot of relevant cytokines showing MFI in the selected clusters (red) compared with MFI across all clusters (black) showing: (i) population of IL-1RA ${ }^{\text {hi }}$ B cells (CD19+) increased in controls and (ii) large population

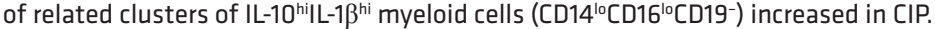

IL-1 $\beta$ release occurs earlier in injury and is decreased by the time our samples are obtained (generally 2 to 3 days at a minimum, after symptom onset). This lack of time resolution in our BALF data may also explain why TNF- $\alpha$ levels were not significantly different. Another explanation is that, although our controls did not have CIP, they underwent bronchoscopy prior to tumor sampling/resection; this bias may be skewing our control IL-1 $\beta$ results. Despite these findings, our IL-12p40 and CXCL-10 (IP-10) data further implicate $\mathrm{CD} 4^{+}$cells in the pathobiology of CIP. IL-12 is a known orchestrator of tissue inflammation and type I polarization. IL-12p40 can form heterodimers with IL-12p70 and IL-23 (26); however, neither of these cytokines was elevated in the BALF of subjects with CIP (Supplemental Table 5). Thus, we postulate that the increased IL-12p40 observed in CIP constitutes the monomeric form. This secreted form has been reported to be 10 - to 20 -fold in excess compared with IL-12p70 in stimulated human peripheral blood cells (27) and has been known to be elevated in patients with asthma during airway inflammation (28). Additionally, IP-10 is known to guide Tcm lymphocytes (a T cell subset seen to be upregulated in our flow cytometry data) to their destination within lymph nodes (29). Therapeutically, antibody-mediated blockade of IL-12p40 and CXCL10 has been used to treat inflammatory diseases $(30,31)$. Our chemotactic cytokine data collectively reflect a lack of neutrophil chemoattraction to the lung (decreased IL-8). Similarly, MIP-3 $\alpha$, which is decreased in patients with CIP BALF, has been previously observed in the context of airway infections (32), is thought to have antimicrobial properties. This observation, along with our IL-8 data and lack of significant neutrophil predominance in our BAL cell differentials (Supplemental Figure 2) further supports the notion that CIP may not be a bacterial infectiontriggered phenomenon. Lastly, our finding of increased CCL17 levels correlates with our flow cytometric finding of increased $\mathrm{CD} 11 \mathrm{~b}^{\text {hi }}$ populations of myeloid cells; $\mathrm{CD} 11 \mathrm{~b}^{+}$cells have been previously identified as a key source of the CCL17-honing chemokine in the lung (33).

Our findings suggest several targets for therapeutic consideration in patients with steroid-refractory CIP. We note upregulation of several TNF- $\alpha^{\text {hi }}$ subsets (lymphoid and myeloid) at baseline in CIP; this finding provides some tissue-specific rationale for the use of infliximab for steroid-refractory CIP, although our BAL cytokine data suggest that timing of TNF- $\alpha$ inhibition may need to be further explored. Importantly, our data also identify several potentially novel populations upregulated in CIP (such as CD62L $\mathrm{L}^{\text {hi }} \mathrm{Tcms}$ and IL-1 $\beta$-expressing monocytes) that could be targeted using existing therapies. Anti-CD62L antibodies or small-molecule inhibitors have been used to attenuate models of lung injury $(34,35)$, although these inhibitors are not currently approved for any clinical indication. Biological agents against IL-1 $\beta$ (e.g., anakinra or canakinumab) are currently either in trials or in use, and thus, further validation of these results could provide the rationale for testing these therapies either 
A

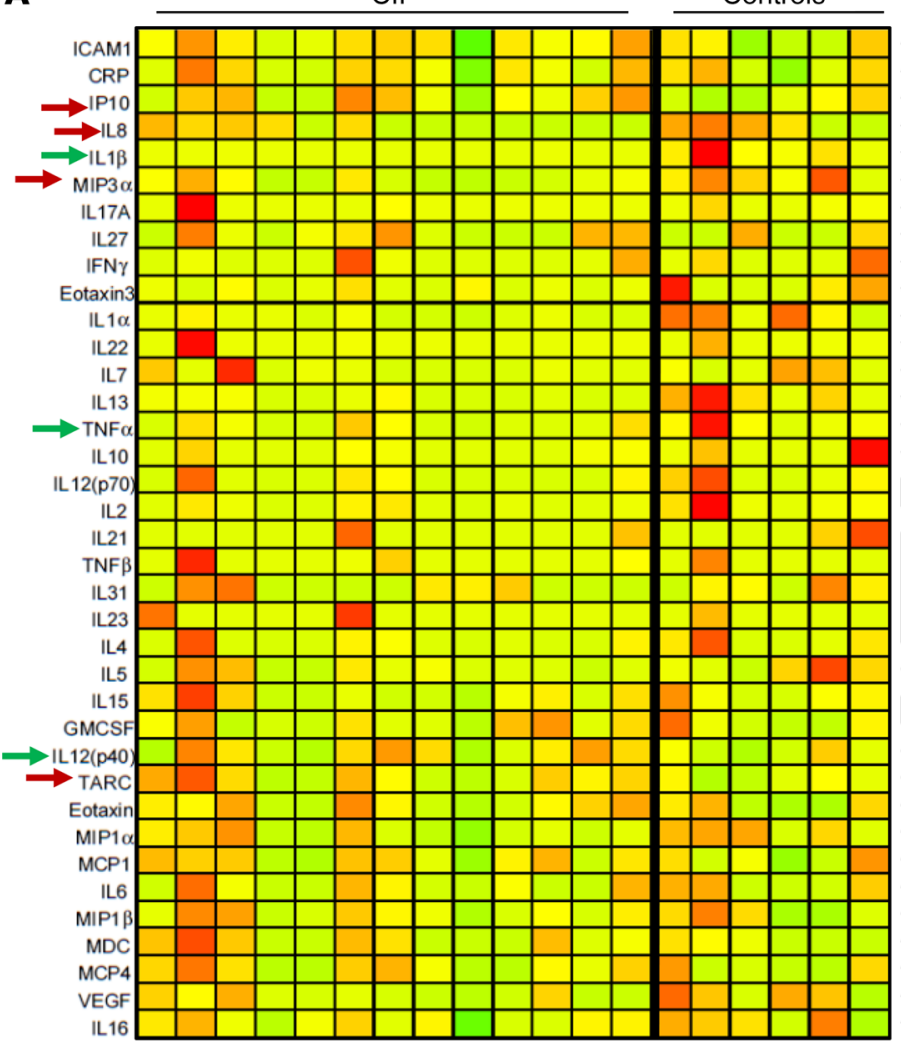

CIP

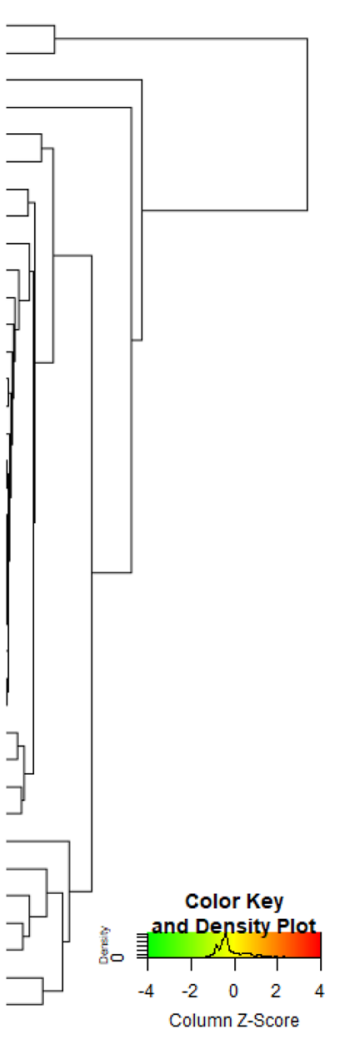

B
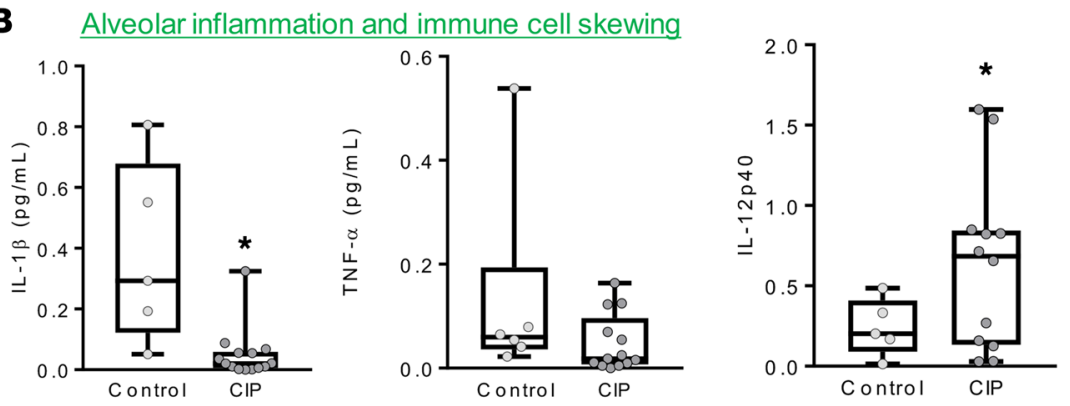

C
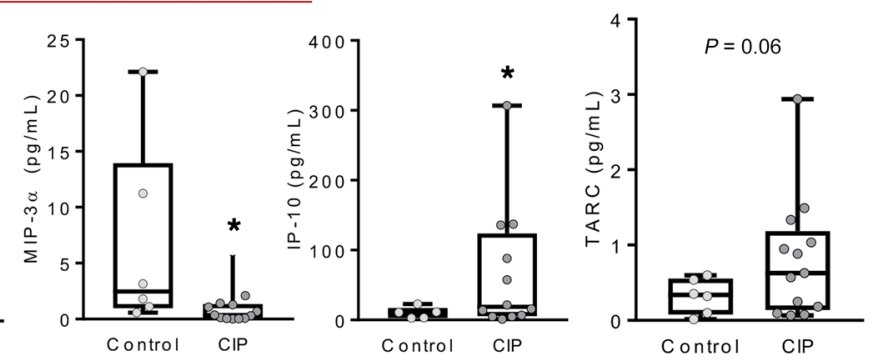

Figure 8. BALF cytokine analysis. (A) Heatmap showing expression of various cytokines in control and CIP BAL supernatant samples. Cytokines are scaled, centered, and hierarchically clustered (using the Euclidean distance). (B) Box-and-whisker plots showing median, minimum, and maximum with individual data point overlay (dots) for select cytokines involved in alveolar inflammation and immune cell skewing (B) or inflammatory cell recruitment/chemotaxis (C). ${ }^{*}$ Denotes significant difference from control BALF samples (Mann-Whitney, $P<0.05$ ).

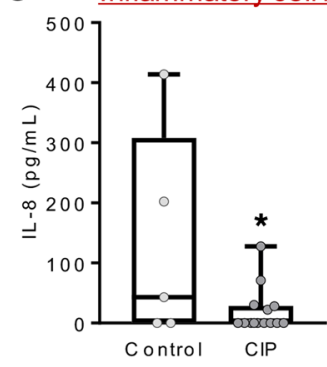

in treating CIP. CCL-17 (TARC) the ligand for CCR4 is usually considered a selective chemoattractant for type 2 cells, although it has been shown to be elevated in sarcoidosis, a classical type I-mediated lung disease (37). Blocking TARC or its receptor CCR4 could decrease $\mathrm{T}$ cell infiltration into the inflamed CIP lungs. Alternatively, transiently enhancing Treg suppressive function could lead to multiple beneficial effects, such as improving control of exuberant type I responses and limiting proliferation, abrogating macrophage proinflammatory responses and ultimately orchestrating lung repair $(22,38)$. For instance, we have previously shown that a shortcourse administration of the DNA methyltransferase inhibitor decitabine can potently augment endogenous Tregs and mediate resolution of lung inflammation and promote lung repair (39). Analysis of CIP rates in ongoing trials utilizing ICI/DNA methyltransferase inhibitor combinations could provide further insight into a potential beneficial effect for these agents from a CIP standpoint.

Although our data provide insight into potential pathobiologic mechanisms in CIP, CIP is unique in comparison to other irAEs regarding incidence (across cancer types) (40) and relationship to overall survival (OS). CIP is much more common in lung cancers compared with other cancers, and although other irAEs have been associated with improved OS, we did not observe a similar association with CIP (13). Thus, we do not believe that our results are necessarily generalizable to other irAEs.

There are several limitations to this study. First, due to the logistical challenges associated with identifying and promptly performing lavage in patients with suspected CIP before antibiotic or steroid administration, our sample sizes are low and thus preclude adjustment for clinical comorbidities (such as chronic obstructive pulmonary disease) that may conas first-line adjuncts or as salvage therapies for high-grade CIP. It is known that transient expression of IL- $1 \beta$ can induce lung inflammation, increase TNF- $\alpha$, and contribute to progressive tissue fibrosis (36); hence, targeting IL-1 $\beta$ could represent an attractive target found our results. Second, while only patients with a negative infectious work-up were included in the CIP cohort, it is possible that BAL cultures did not identify a focus of infection in patients thought to have CIP. Third, although BAL of CIP infiltrates were 


\begin{tabular}{|c|c|c|}
\hline $\mathbf{T}$ cells & $\begin{array}{l}\text { Increased Tcm (expressing } \\
\text { CD62L) and TNF- } a^{\text {hi }} \text {, IFN- } \gamma^{\text {hi }} \\
\text { CD8 } 8^{+} \text {cells }\end{array}$ & $\begin{array}{l}\text { Decreased PD-1/CTLA-4 } \\
\text { expression in Tregs }\end{array}$ \\
\hline \multirow[t]{2}{*}{$\begin{array}{r}\text { Myeloid } \\
\text { cells }\end{array}$} & $\begin{array}{l}\text { Increased CD11b hi, IL-1 } \beta^{\text {hi }} \\
\text { populations }\end{array}$ & $\begin{array}{l}\text { Decreased IL-1RA hi cell } \\
\text { populations }\end{array}$ \\
\hline & $\begin{array}{l}\text { Increase in proinflammatory } \\
\text { cell subsets with increased } \\
\text { expression of endothelial } \\
\text { adhesion markers }\end{array}$ & $\begin{array}{l}\text { Loss of counter-regulatory } \\
\text { suppressive cell phenotypes }\end{array}$ \\
\hline
\end{tabular}

Figure 9. Summary of dysregulated immune cell phenotypes in CIP.

performed in areas not previously affected by tumor, it is possible that presence of malignancy in the nearby airways could have influenced our results.

In conclusion, our data provide several hypothesis-generating insights into the dysregulated alveolar immune dysregulation in patients with CIP. In the absence of a preclinical model for CIP, our findings provide the first rigorous report to our knowledge of immunological mechanisms underlying CIP. In addition to validation in larger clinical cohorts, these data could inform the design of preclinical and translational studies aimed at further understanding the mechanistic basis of CIP, so that targeted therapies can be developed for this morbid complication of immunotherapy.

\section{Methods}

Study population. Patients were enrolled in this prospective observational study if they were (a) diagnosed with NSCLC and (b) treated with ICIs. Patients who received neoadjuvant ICI underwent bronchoscopy with the collection of BALF prior to surgery. Otherwise, BALF was collected whenever patients underwent bronchoscopy. If CIP was suspected, the BALF sampled was categorized as "CIP" if (a) the sample was obtained before initiating steroids and antibiotics and (b) a clinical diagnosis of CIP was adjudicated by the multidisciplinary irTox team (information to follow). After adjudication, patients with CIP were treated with high-dose steroids ( $1 \mathrm{mg} / \mathrm{kg}$ prednisone). Second-line agents (infliximab, i.v. immunoglobulin, or mycophenolate mofetil) were added at the discretion of the treating team if no improvement was noted after 72 hours, as described previously (12).

CIP diagnosis. CIP was defined as (a) shortness of breath, decreased exercise tolerance, exertional desaturation, and/or cough along with (b) the presence of new radiographic infiltrates and (c) lack of evidence of infection (negative cultures on BAL, negative respiratory viral swab) or alternate etiologies (diffuse alveolar hemorrhage, heart failure). Radiographic assessment was performed based on response evaluation criteria in solid tumors (RECIST); cases where the new infiltrates were deemed to represent tumor progression were excluded from both control and CIP groups. A diagnosis of CIP was adjudicated following review and discussion of the pertinent microbiologic and radiographic $(11,12)$ data by the primary oncologist, a second oncologist (JN), 2 pulmonologists (KS, SD), and a radiologist (CTL), with additional input from other members of the immunerelated toxicity team (11) (such as radiation oncology or infectious disease), as needed. Patients in whom clinical equipoise regarding infection was present (e.g., clinical presence of fever, purulent sputum, sick contacts, elevated bands on complete blood count differential) were not adjudicated as CIP even if the BAL cultures were negative.

$B A L$. In control patients, the middle lobe was lavaged. In patients with CIP, an area with new infiltrates not previously known to be associated with tumor was lavaged. The volume of instilled and returned saline was abstracted from the BAL procedure note. BAL specimens were processed with ammonium chloride-potassium lysis solution. Cells were then counted following trypan blue staining to exclude dead cells.

Manual cell differentials. BALF cells were stained with Diff-Quik (Thermo Fisher) and equal numbers of total cells $(n=500)$ were counted per specimen by 2 investigators blinded to the sample group classification as previously described (22).

BAL cytokine measurements. BAL supernatant was collected following centrifugation of the cellular components and stored at $-80^{\circ}$ until further processing. Cytokine measurements were performed using the Mesoscale Discovery platform. Values were normalized to total volume of BAL fluid returned, as noted during bronchoscopy.

Flow cytometry. After thawing samples at $37^{\circ} \mathrm{C}$, cells were stained for flow cytometry. Approximately $1 \times 10^{6}$ cells per sample were stained with violet LIVE/DEAD (Invitrogen). Cells were incubated with human IgG (Rockland Immunochemicals) to block Fc receptors. Cells were then surface stained with BD Biosciences-Pharmingen antibodies: BV510-conjugated anti-CD3 (UCHT1), BUV395-conjugated anti-CD4 (RPA-T4), allophycocyanin-Cy7-conjugated anti-CD25 (M-A251), BUV737-conjugated anti-CD8 (SK1), PE-CF594-conjugated antiCD62L (DREG-56), BV421-conjugated anti-CD127 (HIL-7R-M21), BV650-conjugated anti-CD45RA (HI100), PE-Cy7-conjugated antiCD14 (MoP9), BV711-conjugated anti-PD-1 (EH12), BB700-conjugated anti-CD19 (SJ25C1), PE-Cy7-conjugated anti-CD80 (L307), APC-Cy7conjugated anti-HLD-DR (G46-6), BV650-conjugated anti-CD11b (M1/70), BV711-conjugated anti-C86 (2331-FUN-1), APC-R700-conjugated anti-CD274 (MIH1), BV786-conjugated anti-CD2O6 (19.2), PE-conjugated anti-IL-1RA (AS-17), and BUV395-conjugated antiCD16 (3G8), and intracellularly stained with allophycocyanin-conjugated anti-Foxp3 (PCH101; eBioscience). The following intracellular antibodies from BD Biosciences were also used: Alexa-488-conjugated anti-IL-4 (8D4-8), PE-conujgated anti-IL-10 (JES3-9D7), APC-R700conjugated anti-IL-17A (N49-653), BV605-conjugated anti-IFN- $\gamma$ (B27), BV750-conjugated anti-TNF- $\alpha$ (Mab11), BV786-conjugated anti-CTLA-4 (BNI3), PE-CF594-conjugated anti-TGF- $\beta$ (TW4-9E7), BV510-conjugated anti-IL-8 (G265-8), and BV421-conjugated antiIL-1 (H1b-98; BioLegend). A UV-excitable LIVE/DEAD discrimination assay (Invitrogen) was applied. Cells were then analyzed on a FACSAria (BD Biosciences). Data were analyzed using either FlowJo (TreeStar, Inc.) for traditional gating analyses or R/Bioconductor for unsupervised clustering, as detailed in the information to follow.

$B A L$ cell ex vivo stimulation. Cells were resuspended in a 96-well U-bottom plate using Iscove's modified Dulbecco medium (Thermo Fisher) (10\% heat-inactivated fetal bovine serum, $1 \%$ sodium pyruvate, $1 \%$ HEPES, $2 \mathrm{mM}$ GlutaMax, $100 \mathrm{U} / \mathrm{mL}$ penicillin/streptomycin, and $50 \mu \mathrm{M} \beta$-mercaptoethanol). For lymphocyte stimulation, cells were stimulated with PMA $(40 \mathrm{ng} / \mathrm{mL})$ and ionomycin $(500 \mathrm{ng} / \mathrm{mL})$ for a total for 4 hours, and GolgiStop and GolgiPlug (BD Biosciences) were added the last 3 hours. For myeloid stimulation, cells were stimulated with LPS $(1 \mu \mathrm{g} / \mathrm{mL})$ and IFN- $\gamma(100 \mathrm{ng} / \mathrm{mL})$ for a total of 4 hours, with GolgiStop and GolgiPlug added for the last 3 hours. 
BAL biomarker measurements using Vplex immunoassays. BAL supernatants were used to measure C-reactive protein, eotaxin, eotaxin-3, FGF (basic), GM-CSF, ICAM-1, IFN- $\gamma$, IL- $1 \alpha$, IL-1 $\beta$, IL-2, IL-4, IL-5, IL-6, IL-7, IL-8, IL-8 (HA), IL-10, IL-12 (p40), IL-12 (p70), IL-13, IL-15, IL-16, IL-17A, IL-21, IL-22, IL-23, IL-27, IL-31, IP-10, MCP-1, MCP-4, MDC, MIP- $1 \alpha$, MIP-1 $\beta$, MIP-3 $\alpha$, PlGF, SAA, TARC, Tie-2, TNF- $\alpha$, TNF- $\beta$, VCAM-1, VEGF-A, VEGF-C, VEGF-D, and VEGFR-1/ Flt-1 using Vplex immunoassays (Meso-Scale Discovery), according to the manufacturer's instructions. Samples were run in duplicate. All BAL supernatants were diluted equivalently, the cytokine results were normalized for the amount of BALF recovered, and the results are thus expressed as micrograms per milliliter of recovered BALF, as per the guidelines for measurement of acellular BALF components $(17,41)$.

Statistics. Unsupervised clustering analysis was conducted using the FlowSOM and flowCore packages in R/Bioconductor (42). Briefly, scaled, transformed MFIs for each cell are used as the coordinates for a data point in $n$-dimensional space, where $n$ is the number of fluorophores. A self-organizing map of nodes in this space was to maximize similarity within each node. The distances between nodes reflects the degree of similarity between groups of cells. Importantly, MFI is treated as a continuous variable, thus allowing visualization and analysis of cell subsets where a surface marker expression may be intermediate. Further, because this method of analyzing flow cytometry data incorporates the MFIs for each fluorophore for each cell, it allows for greater resolution of differences in cytokine expression in a multiparametric flow cytometric data set. A graphical abstract of the algorithm is provided in Supplemental Figure 1A. All MFI values are compensated, scaled, and transformed as previously described $(43,44)$. For both $\mathrm{T}$ cells (i.e., singlet, live, $\mathrm{CD}^{+}$cells) and monocytes/B cells (i.e., singlet, live, $\mathrm{CD}^{-}$), the cluster map was first constructed on a concatenated data set composed of unstimulated control, stimulated control, unstimulated CIP, and stimulated CIP samples. This concatenated set represents the sum total of biological replicates ( $n=18 ; 6$ controls and 12 CIP cases). Next, group comparisons between controls and CIP as well as unstimulated and stimulated samples were made by generating group-specific cluster maps as well as a differential cluster map, where a node was considered to be upregulated if there was a greater than $95 \%$ difference between groups. As the initial conditions for the clustering algorithm is randomly chosen, the map shape can differ slightly with each run; each analysis was rerun 5 times to ensure that the same clusters were upregulated across multiple runs (map stability). Lastly, a meta-clustering analysis was performed. This represents an auto-gating strategy where the algorithm attempts to classify cell populations across clusters based on their cytokine profile. The code used to generate the clustering analysis and high-resolution copies of cluster maps are provided in the supplemental data. Scaled, centered values were used to generate the heatmap for BALF cytokine data (gplots package, R). Cytokine profiles were hierarchically clustered (using complete linkage clustering and Euclidean distance). Individual cytokine comparisons were plotted and compared using GraphPad Prism. Two-tailed nonparametric tests (Mann-Whitney) were used to compare mean differences between control and CIP cytokine values. A $P$ value less than 0.05 was accepted as significant.

Study approval. IRB and ethical approval as well as consent was obtained for all participants in this study. All human work was approved by the IRB at the Johns Hopkins Hospital.

\section{Author contributions}

KS was responsible for flow cytometric and multiplex experimental design, data processing and statistical analysis, manuscript writing and review, and figure preparation. JN was responsible for study design, IRB and clinical database design/administration, clinical data analysis, and manuscript writing and review. KS, FD, JN, JRB, SKD, M Shafiq, PMF, JM, MVF, LC, M Soloski, and VA were responsible for study design. KS, FD, QZ, YX, TP, AB, JM, MVF, LC, VA, DSE, KAM, RJK, CLH, BL, JLF, CTL, DFK, ADL, HL, M Shafiq, LY, M Soloski, and EJL were responsible for conducting experiments/adjudication of patient data/data acquisition. QZ, $\mathrm{YX}, \mathrm{TP}, \mathrm{KS}, \mathrm{FD}$, and $\mathrm{AB}$ were responsible for data analysis. KS, $\mathrm{FD}, \mathrm{JN}$, and SKD were responsible for writing the manuscript. All authors reviewed and edited the manuscript.

\section{Acknowledgments}

We wish to thank Naresh Punjabi, Larissa Shimoda, and Mahendra Damarla for their comments on design and interpretation of unsupervised clustering analyses, the Bayview Immunomics Core for their technical expertise with BALF cytokine measurement, and the Bloomberg Kimmel Institute for Cancer Immunotherapy for research and administrative support. This research supported in part by the National Institutes of Health (NIH HL132055, HL131812), Department of Defense (DoDW81XWH-16-1-0510) and the Bloomberg Kimmel Institute for Cancer Immunotherapy.

Address correspondence to: Karthik Suresh, Johns Hopkins Asthma and Allergy Building, 5501 Hopkins Bayview Circle, Baltimore, Maryland 21224, USA. Phone: 410.550.4158; Email: ksuresh2@jhmi.edu.
1. Lipson EJ, Forde PM, Hammers HJ, Emens LA, Taube JM, Topalian SL. Antagonists of PD-1 and PD-L1 in cancer treatment. Semin Oncol. 2015;42(4):587-600.

2. Brahmer J, et al. Nivolumab versus docetaxel in advanced squamous-cell non-small-cell lung cancer. N Engl JMed. 2015;373(2):123-135.

3. Hellmann MD, et al. Nivolumab plus ipilimumab as first-line treatment for advanced non-smallcell lung cancer (CheckMate 012): results of an open-label, phase 1, multicohort study. Lancet Oncol. 2017;18(1):31-41.

4. Balaji A, Verde F, Suresh K, Naidoo J. Pneumonitis from anti-PD-1/ PD-L1 therapy. Oncology (Williston Park, NY). 2017;31(10):739-746, 754.
5. Suresh K, Naidoo J, Lin CT, Danoff S. Immune checkpoint immunotherapy for non-small cell lung cancer: benefits and pulmonary toxicities Chest. 2018;154(6):1416-1423.

6. ARDS Definition Task Force, et al. Acute respiratory distress syndrome: the Berlin Definition. JAMA. 2012;307(23):2526-2533.

7. Khunger M, et al. Incidence of pneumonitis with use of programmed death 1 and programmed death-ligand 1 inhibitors in non-small cell lung cancer: a systematic review and meta-analysis of trials. Chest. 2017;152(2):271-281.

8. Forde PM, Chaft JE, Pardoll DM. Neoadjuvant PD-1 blockade in resectable lung cancer. $N$ Engl J Med. 2018;379(9):e14.
9. Cho JY, et al. Characteristics, incidence, and risk factors of immune checkpoint inhibitor-related pneumonitis in patients with non-small cell lung cancer. Lung Cancer. 2018;125:150-156.

10. Suresh K, et al. Pneumonitis in non-small cell lung cancer patients receiving immune checkpoint immunotherapy: incidence and risk factors. J Thorac Oncol.2018;13(12):1930-1939.

11. Naidoo J, et al. A multidisciplinary toxicity team for cancer immunotherapy-related adverse events. J Natl Compr Canc Netw. 2019;17(6):712-720.

12. Suresh $\mathrm{K}$, et al. Pneumonitis in non-small cell lung cancer patients receiving immune checkpoint immunotherapy: incidence and risk factors. J Thorac Oncol. 2018;13(12):1930-1939. 
13. Suresh K, et al. Impact of checkpoint inhibitor pneumonitis on survival in NSCLC patients receiving immune checkpoint immunotherapy. J Thorac Oncol. 2019;14(3):494-502.

14. Puzanov I, et al. Managing toxicities associated with immune checkpoint inhibitors: consensus recommendations from the Society for Immunotherapy of Cancer (SITC) Toxicity Management Working Group. J Immunother Cancer. 2017;5(1):95.

15. Naidoo J, et al. Pneumonitis in patients treated with anti-programmed death-1/programmed death ligand 1 therapy. JClin Oncol. 2017;35(7):709-717.

16. Tirumani SH, et al. Radiographic profiling of immune-related adverse events in advanced melanoma patients treated with ipilimumab. Cancer Immunol Res. 2015;3(10):1185-1192.

17. Meyer KC, et al. An official American Thoracic Society clinical practice guideline: the clinical utility of bronchoalveolar lavage cellular analysis in interstitial lung disease. Am J Respir Crit Care Med. 2012;185(9):1004-1014.

18. Lucas M, et al. Ex vivo phenotype and frequency of influenza virus-specific CD4 memory T cells. JVirol. 2004;78(13):7284-7287.

19. Agostini C, et al. Involvement of the IP-10 chemokine in sarcoid granulomatous reactions. JImmunol. 1998;161(11):6413-6420.

20. Prasse A, et al. Th1 cytokine pattern in sarcoidosis is expressed by bronchoalveolar $\mathrm{CD} 4^{+}$and $\mathrm{CD} 8^{+}$ T cells. Clin Exp Immunol. 2000;122(2):241-248.

21. Yamasaki H, Ando M, Brazer W, Center DM, Cruikshank WW. Polarized type 1 cytokine profile in bronchoalveolar lavage $\mathrm{T}$ cells of patients with hypersensitivity pneumonitis. JImmunol. 1999;163(6):3516-3523.

22. D'Alessio FR, et al. Resolution of experimental lung injury by monocyte-derived inducible nitric oxide synthase. JImmunol. 2012;189(5):2234-2245.
23. Gianchecchi E, Fierabracci A. Inhibitory receptors and pathways of lymphocytes in the role of PD-1 in Treg development and their involvement in autoimmunity onset and cancer progression. Front Immunol. 2018;9:2374.

24. Rowshanravan B, Halliday N, Sansom DM. CTLA-4: a moving target in immunotherapy. Blood. 2018;131(1):58-67.

25. Lopez-Castejon G, Brough D. Understanding the mechanism of IL-1 $\beta$ secretion. Cytokine Growth Factor Rev. 2011;22(4):189-195.

26. Cooper AM, Khader SA. IL-12p40: an inherently agonistic cytokine. Trends Immunol. 2007;28(1):33-38.

27. D'Andrea A, et al. Production of natural killer cell stimulatory factor (interleukin 12) by peripheral blood mononuclear cells. JExp Med. 1992;176(5):1387-1398.

28. Walter MJ, Kajiwara N, Karanja P, Castro M, Holtzman MJ. Interleukin $12 \mathrm{p} 40$ production by barrier epithelial cells during airway inflammation. J Exp Med. 2001;193(3):339-351.

29. Sung JH, et al. Chemokine guidance of central memory $\mathrm{T}$ cells is critical for antiviral recall responses in lymph nodes. Cell. 2012;150(6):1249-1263.

30. Mannon PJ, et al. Anti-interleukin-12 antibody for active Crohn's disease. N Engl JMed. 2004;351(20):2069-2079.

31. Mayer L, et al. Anti-IP-10 antibody (BMS936557) for ulcerative colitis: a phase II randomised study. Gut. 2014;63(3):442-450.

32. Starner TD, Barker CK, Jia HP, Kang Y, McCray PB. CCL2O is an inducible product of human airway epithelia with innate immune properties. Am J Respir Cell Mol Biol. 2003;29(5):627-633.

33. Medoff $\mathrm{BD}$, et al. CD11b $\mathrm{b}^{+}$myeloid cells are the key mediators of Th2 cell homing into the airway in allergic inflammation. JImmunol. 2009;182(1):623-635.
34. Ridings PC, et al. A dual-binding antibody to E- and L-selectin attenuates sepsis-induced lung injury. Am JRespir Crit Care Med.1995;152(1):247-253.

35. Seekamp A, Regel G, Rother K, Jutila M. The effect of anti-L-selectin (EL-246) on remote lung injury after infrarenal ischemia/reperfusion. Shock. 1997;7(6):447-454.

36. Kolb M, Margetts PJ, Anthony DC, Pitossi F, Gauldie J. Transient expression of IL-1beta induces acute lung injury and chronic repair leading to pulmonary fibrosis. JClin Invest. 2001;107(12):1529-1536.

37. Heffner DK. Treatments for pulmonary sarcoidosis. Respir Med. 2008;102(11):1674.

38. Mock JR, et al. Foxp $3^{+}$regulatory $\mathrm{T}$ cells promote lung epithelial proliferation. Mucosal Immunol. 2014;7(6):1440-1451.

39. Singer BD, et al. Regulatory T cell DNA methyltransferase inhibition accelerates resolution of lung inflammation. Am J Respir Cell Mol Biol. 2015;52(5):641-652.

40. Puzanov I, et al. Managing toxicities associated with immune checkpoint inhibitors: consensus recommendations from the Society for Immunotherapy of Cancer (SITC) Toxicity Management Working Group. JImmunother Cancer. 2017;5(1):95.

41. Haslam PL, Baughman RP. Report of ERS Task Force: guidelines for measurement of acellular components and standardization of BAL. Eur Respir J. 1999;14(2):245-248.

42. R Core Team. R: A language and environment for statistical computing. R Foundation for Statistical Computing, Vienna, Austria. http:// www.R-project.org/. Accessed July 11, 2019.

43. Hahne F, et al. flowCore: a Bioconductor package for high throughput flow cytometry. BMC Bioinformatics. 2009;10:106.

44. Van Gassen S, et al. FlowSOM: using self-organizing maps for visualization and interpretation of cytometry data. Cytometry. 2015;87(7):636-645. 